\title{
Continuous-time model and physical simulation of population dynamics of sickle cell anaemia
}

\author{
O. O. Akanbi ${ }^{1,2}$, S. O. Edeki ${ }^{2, *}$, O. A. Agbolade ${ }^{1,2}$ \\ ${ }^{1}$ Department of Mathematics \& Statistics, Federal Polytechnic Ilaro, Ilaro, Nigeria \\ ${ }^{2}$ Department of Mathematics, Covenant University, Ota, Nigeria
}

\section{A R T I C L E IN F O}

\section{Article history:}

Received 11 December 2016

Received in revised form

15 March 2017

Accepted 27 April 2017

\section{Keywords:}

Renewal equation

Hardy-weinberg equation

Simulation mating pattern

Sicklers

Interaction function

\section{Introduction}

Individual genotype AA, AS, SS, SC or AC differs amongst the world's population (Saladin and Miller, 1998). Of interest however, is the fact that genetic mechanism on morphogenetic traits is still not clearly understood as it is seen to occur with variable frequency in different populations and thus useful in evaluating and analyzing evolutionary forces and classification (Das and Sengupta, 2003). Meanwhile, marked inter-individual variability in genetic and non-genetic factors has been said to possess the ability to influence the disposition of many endobiotics and xenobiotic affecting health (Lamba et al., 2002).

SCA which is a genetically transmitted disease is caused by a defective allele (mutant form) of the gene coding for a sub unit of the haemoglobin protein. The Sickle haemoglobin tends to precipitate or clump together within the red blood cells after releasing its oxygen. If the clumping is extensive the red blood cell assumes an abnormal sickle shape. These sickle red blood cells plug the blood vessels thus preventing normal red blood cell passage and consequently depriving the tissue of needed oxygen.

\footnotetext{
* Corresponding Author.

Email Address: soedeki@yahoo.com (S. O. Edeki)

https://doi.org/10.21833/ijaas.2017.06.002

2313-626X/C 2017 The Authors. Published by IASE.

This is an open access article under the CC BY-NC-ND license

(http://creativecommons.org/licenses/by-nc-nd/4.0/)
}

Each person has two copies of the gene that determines whether that person has Sickle Cell Anaemia. If both copies are normal alleles then only normal haemoglobin is produced AA. If one of the two alleles is defective then that person has a mixture of normal and Sickle haemoglobin: a condition known as Sickle Cell trait "AS" (Carrier). If both alleles are defective, essentially only sickle haemoglobin is made and the person has Sickle Cell Anaemia "SS".

The first case of SCA was reported in 1910 on a Jamaican student in the USA (Herrick, 2014). The term SCA is a term first used by Mason (1922) to describe the homozygous state (Serjeant and Serjeant, 1992). Neel (1949) illustrated that SCA was transmitted as a recessive gene ' $\mathrm{S}$ '. But it is well known by scientist now that the gene is neither dominant nor recessive but of intermediate penetrance.

The allele causing SCA is found most often in people of African ancestry. It was traced to one family in Ghana (krobo people) in 1670 . The "S" gene is found mainly where malaria is endemic. It also occurs in people of Mediterranean, Arab, East India, South and Central American ancestry. From the world population of about 7,058, 157,073 (Umoh et al., 2010). (Number of people officially counted). 5\% of world population lives with SCD (Lamba et al., 2002). Mathematically, about $352,907,854$ people have haemoglobin disorder "officially counted" while every year 300,000 infants are born with SCD, including 200,000 cases in Africa (NIH, 2014). 
Nigeria of about 150 million populations with growth rate of $3.2 \%$ has prevailing rate of 150,000 offspring per year. Nigeria by the virtue of her population ranks first as a SCA endemic country in Africa with annual infant deaths totally around $100,000,8 \%$ of infant mortality. This alarming infant death related to SCA made the authors to carryout mathematical population dynamics.

Mathematical models of heredity are largely based on one-locus, two allele gene populations, where little or no attempt is made to consider the dynamics of the population and the analysis is somehow probabilistic, accounting of genotype composition of a diploid population. Nevertheless, success approaches have been developed for continuous-time model that laid more emphasis on the dynamics of population of SCA (Tchuenche, 2002). Rosen (1983) presented a mathematical model for polygamous system, where he introduced the interaction function (KMF) where $\mathrm{M}$ and $\mathrm{F}$ represent the male and the female population sizes respectively. A new set of functional differential equations modeling heterosexual population dynamics with $\mathrm{K}$ as the polygamy factor was developed by Sowunmi (1993); there, he proved that population renewal is the product of an interaction between the reproductive males and the nongestating reproductive females. Sowunmi (1993), Tchuenche (2003) and Tchuenche (2007) were the first to use the general interaction function $F_{i j}$ where $F_{i j}$ contributes the major mathematical background for the formulation of birth renewal equation. Tchuenche $(2003,2007)$ has done a lot in the dynamics of SCA. He is the pioneer mathematician who made use of differential equation to model the disease (SCA). Tchuenche (2002) demonstrated the dynamical behaviour of SCA by a set of first-order non-linear partial differential equation. He also developed the birth renewal equation the same year. The age and character-dependent population dynamics model of genetically transmitted diseases where standard techniques of functional analysis were employed to further simplify and solve the basic equations implicitly in special cases of SCA was introduced (Tchuenche, 2005). Another satisfactory progress on SCA was the adequate mathematical picture of the pattern of inheritance of the allele " $\mathrm{S}$ ". Several models had provided solution to the following:

- How well do bounds capture behavioural features such as long term persistence of the diseases?

- In which parameter does the model perform best and how relevant are they to the real life situation?

Migration which is an important demographic parameter has been considered in terms of modeling SCA and polygamy. Another beauty of population dynamics of the genetically transmitted disease (SCA) which takes age, mating behaviour and a physiological factor into account was proposed in the Theoretical Population Dynamics Model (TPDM) of a generically transmitted disease

(Tchuenche, 2007).

Liddell et al. (2014) made it critically clear with his mathematical model of sickle cell genome frequency in response to selective pressure from malaria that selection pressure for the carrier gene in the presence of increase in malaria death for either adult or children has higher frequencies of the gene as well as shortened time to reach these frequencies.

Recently, differential impact of sickle cell trait on symptomatic and asymptomatic malaria was introduced where a decreased frequency of S-gene may eventually increase the overall prevalence of both symptomatic and asymptomatic malaria. Hence, the control of symptomatic malaria might result in evolutionary repercussion, despite short-term epidemic logical benefits Shim et al. (2012).

\section{Modeling background}

A population is often regarded as a group of individuals that can cross (mate) and give birth to offspring (neonates). Human population differs with respect to their genotype, age, Rhesus factor, physiology etc. Hence, they can be classified according to their distinguishable differences. The following assumptions are taken into consideration:

i. The entire population has an age structure that spans through where is the life span.

ii. A genotype structure composed of three distinct genetic subgroups is duly imposed on the population i.e. normal AA, carriers AS, and sickles SS.

iii. Monogamy form of family setting is also assumed.

iv. The spread of the population is spatially homogeneous.

v. Variables are the independent variables: Age and time respectively. Consider below in Table 1.

Table 1: Genotype, sex, and phenotype

\begin{tabular}{cccc}
\hline \multirow{2}{*}{ Genotype } & \multicolumn{2}{c}{ Sex } & \multirow{2}{*}{ Phenotype } \\
\cline { 2 - 3 } & Females & Males & \\
\hline AA & $f_{1}$ & $m_{1}$ & Normal \\
AS & $f_{2}$ & $m_{2}$ & Carriers \\
SS & $f_{3}$ & $m_{3}$ & Sicklers \\
\hline
\end{tabular}

Suffixes $i=1, j=2, k=3$ correspond to $A A=1, A S=2$, $\mathrm{SS}=3$ respectively.

Where $f_{i}(x, y) \geq 0$ represents the population density of females in group $i$ with age $x$ at time $y$, $m_{i}(x, y) \geq 0$ represents the population density of males in group $i$ with age $x$ at time $y$, and $F_{i j}\left\{\left[m_{i}(x, y): f_{i}\left(x^{1}, y\right)\right], x, x^{1}, y\right\}$ is the function governing the interaction between males of class $i$ males and females of class $\mathrm{j}$.

Also, $\delta_{i j}^{k}$ is the probability of getting a neomate of class $i$ from mating between class $i$ males and class $j$ female.

$\Gamma$ - the probability of acquiring the $S$ gene from any of the parents. 
$f_{j}^{i}$-number of females of class i interacting with class $j$ males (referred to as number of couples) in a monogamous setting.

(a) $F_{i j}\left\{\left[\left(f_{i ; 2}, m_{2}, m_{3}\right) \alpha, \alpha^{1}, y\right]\right\}:$ In this case, every females at reproductive age has just a life partner and vice-versa.

(b) $F_{i j}\left\{\left[\left(f_{i ;}, f_{1}, f_{2}, f_{3}\right) \alpha^{1}, \alpha, y\right]\right\}$ : This means that some males of class i interact with normal females $f$, others with females carriers $f_{2}$ and the remaining with sicklers $f_{3}$. In this case, polygamy may arise. In the sequel, we shall refer only to the former.

We cannot affirm with certainty that a woman aged $x$ will marry a man aged $x^{1}$. This is a problem that takes into cognizance numerous factors that cannot be controlled mathematically. We have therefore modified the interaction function $F_{i j}$ to suit our case where the population is subdivided into three groups; namely, AA, AS, and SS. Our two-sex mixing function $F_{i j}$ can be defined as:

$$
F_{i j}\left\{\left[\left(f_{i}(\alpha, y) ; m_{j},\left(\left(\alpha^{1}, y\right)\right]\right) \alpha^{1}, y ; \alpha, \alpha^{1}, y\right\}\right.
$$

where, $F_{i j}$ is a positive real-valued function and has compact support with respect to $\left(\alpha^{1}, y\right)$. The reproductive age of females spans the interval $\left[w_{f_{r}}, w_{f_{s}}\right]$ and $\left[w_{m_{r}}, w_{m_{s}}\right]$ for males. Hence;

$\operatorname{SupF}_{i j} \leq\left[w_{f_{r}}, w_{f_{s}}\right] \times\left[w_{m_{r}}, w_{m_{s}}\right]$.

\section{Results of the different mating patterns}

The techniques employed to derive the results of mating placed more emphasis on the individuals than the genetics.

The possible outcome of the interactions could produce (Akanbi, 2006). Normal individuals:

$F_{11}\left[\left(f_{1} ; m_{1}\right), \alpha, \alpha^{1}, y\right]$,

$F_{12}\left[\left(f_{1} ; m_{2}\right), \alpha, \alpha^{1}, y\right]$ and

$F_{22}\left[\left(f_{2} ; m_{2}\right), \alpha, \alpha^{1}, y\right]$

Carriers:

$F_{12}\left[\left(f_{1} ; m_{2}\right), \alpha, \alpha^{1}, y\right]$

$F_{13}\left[\left(f_{1} ; m_{3}\right), \alpha, \alpha^{1}, y\right]$,

$F_{22}\left[\left(f_{2} ; m_{2}\right), \alpha, \alpha^{1}, y\right]$, and

$F_{23}\left[\left(f_{2} ; m_{3}\right), \alpha, \alpha^{1}, y\right]$

Sicklers:

$F_{22}\left[\left(f_{2} ; m_{2}\right), \alpha, \alpha^{1}, y\right]$,

$F_{23}\left[\left(f_{2} ; m_{3}\right), \alpha, \alpha^{1}, y\right]$, and

$F_{33}\left[\left(f_{3} ; m_{3}\right), \alpha, \alpha^{1}, y\right]$

The probabilities of acquiring the gene from either parents are the same, this point is worth noting, because this is what implies $F_{i j}=F_{j i}$.

\subsection{The corresponding birth equations}

For $x \in(0, L] 0 \leq \delta_{i j}^{k} \leq 1$ and the compactness of the support of $F_{i j}$ : the birth equations are given below (Eqs. 1, 2, and 3):

$$
\begin{aligned}
& B_{1}(y)=\iint_{00}^{\infty \infty}\left(H_{1}\right) d \alpha d \alpha^{1} \\
& B_{1}(y)=\iint_{00}^{\infty \infty}\left(H_{2}\right) d \alpha d \alpha^{1}
\end{aligned}
$$

$B_{1}(y)=\iint_{00}^{\infty} \infty\left(H_{3}\right) d \alpha d \alpha^{1}$

where,

$H_{1}=\delta_{11}^{(1)} F_{11}+\delta_{12}^{(1)} F_{12}+\delta_{22}^{(1)} F_{22}$,

$H_{2}=\delta_{12}^{(2)} F_{12}+\delta_{13}^{(2)} F_{13}+\delta_{22}^{(2)} F_{22}+\delta_{23}^{(2)} F_{23}$,

$H_{3}=\delta_{22}^{(3)} F_{22}+\delta_{23}^{(3)} F_{23}+\delta_{33}^{(3)} F_{33}$

Such that:

$\beta(y)=\sum_{n=1}^{3} B_{n}(y)$

\subsubsection{Properties of $B_{n}(y)$}

i $B_{n}(\boldsymbol{y})$ is well defined and non-negative $F_{i j} \geq$ 0 . Human beings are good examples of birth flow population in which birth occur continuously over the time interval.

ii $B_{n}(\boldsymbol{y})$ is a first degree homogeneous function of its arguments i.e. $B_{n} K F_{i j}(y)=K B_{n} F_{i j}(y)$

iii $B_{n}(\boldsymbol{y})=0$ for any population in which either male or female is absent.

\subsection{Physical simulation of the model}

This will help to clarify the biological process behind the mathematical model. To reduce the complexity of the generation gene pool, two alleles namely $\mathrm{HbA}$ (normal allele) and $\mathrm{HbS}$ (sickle cell allele) haemoglobin of males and non-gestating reproductive females were only taken into account.

We used beads of different colours to represent alleles. The births are simulated by randomly drawing beads from the gene pool. For each generation number of individuals with each of three possible allele combination (namely AA, AS, SS are obtained $F_{i j}=F_{j i}$ i.e. AS $=\mathrm{SA}$ are indistinguishable) were drawn.

\subsubsection{Assumptions of the model}

1. All members of the population neglect celibacy as a way of life.

2. Immigration or Emigration is not allowed.

3. The population is a non-gestating female dominant system.

4. Every member of the population is within the reproductive age.

According to Adeyinka Sickle Cell Hope Alive Foundation (SCHAF), "Nigeria has about 5 million people with SS genotype, 40 million with AS genotype and about $66-72 \%$ with AA genotype" $(\mathrm{NIH}, 2014)$. Mathematically, Nigeria accounts for $3 / 4$ of people living with SCD globally.

Hence, the generation gene pool constitutes alleles which replicate the genotypic population of Nigeria. The population size of the gene pool is taken to be 3136 alleles. This represents the initial genetic make-up of reproductive non-gestating female and male adults. Hence, in the gene pool, we have $2640 \mathrm{~A}$ 
alleles and $496 \mathrm{~S}$ alleles. That is, 1568 reproductive couples are considered.

Let $P$ be the frequency of the allele " $(\mathrm{A})$ " in the population and let $q$ be the frequency of the allele " $\mathrm{S}$ " in the population. By Hardy-Weinberg equation $(P+$ $q)^{2}=1$, we obtained the frequency of each generation (Table 2). From the Table 2, we observe that an average of $70 \%$ neonates will be $A A, 27 \% A S$ and $3 \% S S$. Data in Table 2 is graphed in Fig. 1 for analysis.

Table 2: Trial and frequency

\begin{tabular}{ccccccc}
\hline \multirow{2}{*}{ Generation/trial } & \multicolumn{3}{c}{ AA } & \multicolumn{2}{c}{ SS } \\
\cline { 2 - 6 } & No. & Frequency & No. & Frequency & No. & Frequency \\
\hline 1 & 1111 & $69 \%$ & 412 & $28 \%$ & 45 & $3 \%$ \\
2 & 1073 & $75 \%$ & 468 & $23 \%$ & 27 & $2 \%$ \\
3 & 1021 & $70 \%$ & 504 & $27 \%$ & 43 & $3 \%$ \\
4 & 1071 & $68 \%$ & 450 & $29 \%$ & 47 & $3 \%$ \\
5 & 1112 & $70 \%$ & 415 & $27 \%$ & 41 & $3 \%$ \\
6 & 1116 & $70 \%$ & 410 & $27 \%$ & 42 & $3 \%$ \\
7 & 1113 & $69 \%$ & 411 & $28 \%$ & 44 & $3 \%$ \\
\hline \multicolumn{7}{c}{ Domain $=\{p: 0 \leq p \leq 1\}$ and Range $=\{B: 0 \leq B \leq 1568\}$}
\end{tabular}

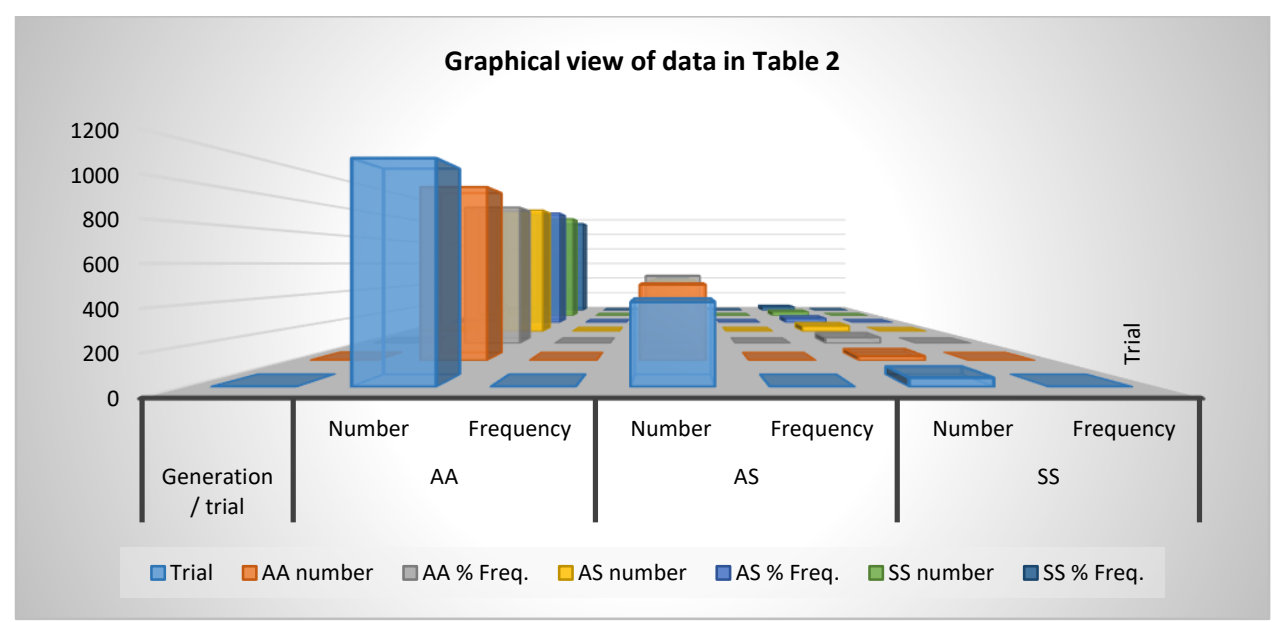

Fig. 1: Data in Table 2 for data analysis purposes

Also, Reader(s) are referred to NHLBI (2015) and Akanbi et al., (2016) for detail of Blood-sample of normal RBC, and Blood-sample of sickled RBC while Table 1 for Fig. 1.

\section{Concluding remark}

Considering the integral Eqs. 1, 2, and 3 where relative activities between AS \& AS, AS \& SS, and SS \& SS are not zero; the birth of sicklers is a continuous process, which may likely increase over time. Even if genotype screening before marriage is imposed, the population of sicklers is not likely to reduce at the long run.

From the physical simulation of population size of 3136 alleles with $2640 \mathrm{~A}$ alleles and $496 \mathrm{~S}$ alleles in the gene pool, the emergence of sicklers (SS) still stands the chance of an average of $3 \%$ for seven trials/generations. The implication of this is that there is great possibility for the population of SS to increase over time. Though, bone marrow transplants are a milestone as a curative measure to SCA. But it is too expensive for a common man. Hence, we suggest that a cheaper curative measure still stands an area of research that must not be neglected.

\section{Acknowledgement}

The authors are grateful to Covenant University for financial support.

\section{Conflict of interest}

There is no an ethical issue or conflict of interest regarding the publication of this paper.

\section{References}

Akanbi OO (2006). Discrete and continuous-time models of the genetic structure of Sickle-Cell Anaemia. M.Sc. Thesis, University of Ibadan, Ibadan, Nigeria.

Akanbi 00, Edeki SO, and Agbolade OA (2016). Monte carlos simulation approach to population dynamics of sickle cell anaemia. American Journal of Applied Sciences, 14(3): 358364.

Das B and Sengupta SA (2003). Note on some morphogenetics variables among the Sonowal Kacharis of Assam. Antropologist, 5(3): 211-212.

Herrick JB (2014). Peculiar elongated and sickle-shaped red blood corpuscles in a case of severe anemia. Jama, 312(10): 10631063.

Lamba JK, Lin YS, Thummel K, Daly A, Watkins PB, Strom S, Zhang J, and Schuetz EG (2002). Common allelic variants of cytochrome and their prevalence in different populations. Pharmacogenetics and Genomics, 12 (2): 121-132.

Liddell C, Owusu-Brackett N, and Wallace D (2014). A mathematical model of Sickle cell genome frequency in response to selective pressure from malaria. Bulletin of Mathematical Biology, 76(9): 2292-2305.

Mason VR (1922). Sickle cell anemia. Journal of the American Medical Association, 79(16): 1318-1320.

Neel JV (1949). The inheritance of sickle cell anemia. Science, 110(2846): 64-66. 
NHLBI (2015). What Is Sickle Cell Disease?. National Heart, Lung, and Blood Institute. Available online at: http://www.nhlbi.nih.gov/health/health-topics/topics/sca.

NIH (2014). What Is Sickle Cell Disease?. National Heart, Lung, and Blood Institute. Available online at: https://www.nhlbi.nih gov/health/health-topics/topics/sca/

Rosen KH (1983). Mathematical models for polygamous mating systems. Mathematical Modelling, 4(1): 27-39.

Saladin KS and Miller L (1998). Anatomy and physiology. McGrawHill, New York, USA.

Serjeant GR and Serjeant BE (1992). Sickle cell disease. Oxford University Press, Oxford, UK.

Shim E, Feng Z, and Castillo-Chavez C (2012). Differential impact of sickle cell trait on symptomatic and asymptomatic malaria. Mathematical Biosciences and Engineering, 9(4): 877-898.

Sowunmi COA (1993). A model of Heterosexual population Dynamics with Age structure and gestation period. Journal of Mathematical Analysis and Applications, 172(2): 390-411.
Tchuenche JM (2002). Mathematical population Dynamics of Sickle-Cell Anaemia. Ph.D. Dissertation, University of Ibadan, Nigeria, West Africa.

Tchuenche JM (2003) Classroom notes: A note on the MacKendrick Von Foerster type model in a population with genetic structure. International Journal of Mathematical Education in Science and Technology, 34(3): 463-469.

Tchuenche JM (2005). Realistic patterns of inheritance of SickleCell Anaemia Gene: A theoretical Approach. Journal of Biological System, 13(1): 13-22.

Tchuenche JM (2007). Theoretical population dynamics model of a genetically transmitted disease: Sickle-cell anaemia. Bulletin of Mathematical Biology, 69(2): 699-730.

Umoh AV, Abah GM, Ti E, and Essien EM (2010). Haemoglobin genotypes: A prevalence study and implications for reproductive health in Uyo, Nigeria. Nigerian Journal of Medicine, 19(1): 36-41. 